\title{
Polarization dependence of the absorption coefficient for an array of strained quantum wires
}

\author{
Igor Vurgaftman and Jasprit Singh \\ Solid State Electronics Laboratory, Department of Electrical Engineering and Computer Science, \\ The University of Michigan, Ann Arbor, Michigan 48109-2122
}

(Received 24 October 1994; accepted for publication 17 January 1995)

\begin{abstract}
The polarization dependence of the absorption coefficient for compressively and tensilely strained quantum wires is investigated as a function of strain, the lateral wire width, and the ratio between the wire and barrier widths. The results are markedly different from the case of an unstrained quantum wire and exhibit a significant variation with the sign and amount of built-in strain. In the case of compressive strain, absorption of light polarized in the growth direction is considerably suppressed near the band edge, while the ratio between the absorption coefficients for light polarized parallel and perpendicular to the wire is determined by the relative prominence of strain and quantum confinement effects. A discussion of how these results can be applied to characterization of the achieved quantum confinement by polarization-resolved photoluminescence spectroscopy is given. () 1995 American Institute of Physics.
\end{abstract}

\section{INTRODUCTION}

Semiconductor quantum wires are being actively pursued by a number of research groups for their potential applications in optoelectronics and studies of low-dimensional physics. ${ }^{1-3}$ Owing to the formidable challenges encountered in the quantum wire fabrication, it is important to identify characterization tools allowing one to judge the quality of the structures. Polarization-dependent photoluminescence (PL) and PL excitation (PLE) spectroscopies have emerged as important characterization technologies capable of providing feedback on structural integrity. ${ }^{3,4}$ The marked difference of the polarization dependence of PL in quantum wells and wires clearly demonstrates whether the quasi-one dimensional (1D) confinement of electrons and holes has been attained. Thus in quantum wires, one gets a fourfold enhancement in the signal for light polarized along the wire axis as compared to that for light polarized perpendicular to it. ${ }^{5,6}$ However, this is true only for unstrained cylindrical or rectangular quantum wires. The situation is quite different in strained quantum wires since strain is known to alter the symmetry of band edge states. Since considerable interest in strained structures exists, it is important to identify how the polarization dependence changes as a function of strain and wire dimension.

In this article, we calculate the polarization dependence of the absorption coefficient in a variety of compressively and tensilely strained quantum wires. The approach developed for unstrained quantum wires of an arbitrary cross section ${ }^{4}$ must be supplemented with the deformation potential theory ${ }^{7}$ to yield the complete Hamiltonian and its eigenvalues and eigenfunctions. In this article, we investigate the near-band edge polarization dependence of the absorption coefficient in compressively and tensilely strained quantum wires by a variational calculation of the strain tensor, using the latter in the deformation potential formalism, and solving the Kohn-Luttinger Hamiltonian with the appropriate terms due to strain by a finite difference approximation in the cross sectional plane of the quantum wire. Exciton absorption is neglected in this discussion.

\section{FORMALISM}

We will confine our discussion to an infinite 1D array of quantum wires grown on a (001) GaAs substrate to a width $W=80 \AA$ in the $x$ direction (neglecting deviations at the edges for any practical array). The lateral confinement is achieved by etching and regrowth of a higher-band gap material in the $y$ direction. For compressively and tensilely strained quantum wires, the well material is $\mathrm{In}_{x} \mathrm{Ga}_{1-x}$ As and $\mathrm{GaAs}_{1-x} \mathrm{P}_{x}$, respectively. In order to reduce the number of variables in the problem, we keep the band gap discontinuity constant at $\approx 0.25 \mathrm{eV}$ (60\%:40\% distribution between the conduction and valence band, respectively) by taking $\mathrm{Al}_{y} \mathrm{Ga}_{1-y} \mathrm{As}$ barriers with the properly adjusted value of $y$. This assumption does not affect our most significant results, since the relative polarization dependence of the band edge transitions is more or less independent of the barrier height. In the geometry used by us, the wire axis is along the $z$ direction. The characteristic dimensions of the problem are the lateral wire width $d_{w}$ and the barrier width $d_{b}$ (along the $y$ axis). Assuming that the barrier material is lattice matched to the substrate, the objective is to find the form of the strain tensor that minimizes the elastic energy for a single period of the infinite array. While, in general, the strain tensor calculation for this geometry is tedious, we adopt simple approximations with the correct functional form in the familiar limits. By the assumption of no dislocations in the strained layer, the in-plane diagonal components of the strain tensor $\epsilon_{y y}$ and $\epsilon_{z z}$ are equal and determined only by the mismatch in the lattice constants of the substrate and layer materials:

$$
\epsilon_{y y}=\epsilon_{z z}=\epsilon=\frac{\alpha_{S}}{\alpha_{L}}-1
$$


Assuming that the only nonzero components of the strain tensor are diagonal, we minimize the expression for the elastic energy of a single period of a cubic system: ${ }^{7}$

$$
\begin{aligned}
U= & W d_{w}\left[\frac{1}{2} c_{11}\left(\epsilon_{x x}^{2}+\epsilon_{y y}^{2}+\dot{\epsilon}_{z z}^{2}\right)\right. \\
& \left.+c_{12}\left(\epsilon_{y y} \epsilon_{z z}+\epsilon_{z z} \epsilon_{x x}+\epsilon_{x x} \epsilon_{y y}\right)\right]+W d_{b}\left[\frac{1}{2} c_{11}\left(\epsilon_{x x}^{2}\right)\right]
\end{aligned}
$$

for the unknown $\epsilon_{x x}$ component. The result is

$$
\epsilon_{x x}=-\frac{2 c_{12}}{c_{11}} \frac{d_{w}}{d_{w}+d_{b}} \epsilon
$$

This reduces to the expression for the strained quantum wells ${ }^{7}$ in the limit $d_{b} \rightarrow 0$. Note also that for a very small aspect ratio $r=d_{w} /\left(d_{w}+d_{b}\right), \epsilon_{x x} \rightarrow 0$. This derivation presumes that the lateral barrier material is the same as the vertical barrier material. A strain tensor equivalent to that in the quantum well case is obtained if the lateral barrier material has the same lattice constant as the quantum wire region. Using the deformation potential formalism for the states of angular momentum $|3 / 2, \pm 3 / 2\rangle$ and $|3 / 2, \pm 1 / 2\rangle$ near the top of the valence band, the only nonzero matrix elements of the strain Hamiltonian $H_{\epsilon}$ are

$$
\begin{aligned}
\langle 3 / 2, & \left. \pm 3 / 2\left|H_{\epsilon}\right| 3 / 2, \pm 3 / 2\right\rangle \\
= & a\left(\epsilon_{x x}+\epsilon_{y y}+\epsilon_{z z}\right)+\frac{b}{2}\left(\epsilon_{x x}+\epsilon_{y y}-2 \epsilon_{z z}\right), \\
& =a\left(2-\frac{2 c_{12}}{c_{11}} \frac{d_{w}}{d_{w}+d_{b}}\right) \epsilon-\frac{b}{2}\left(1+\frac{2 c_{12}}{c_{11}} \frac{d_{w}}{d_{w}+d_{b}}\right) \epsilon, \\
\langle 3 / 2, & \left. \pm 1 / 2\left|H_{\epsilon}\right| 3 / 2, \pm 1 / 2\right\rangle \\
= & a\left(\epsilon_{x x}+\epsilon_{y y}+\epsilon_{z z}\right)-\frac{b}{2}\left(\epsilon_{x x}+\epsilon_{y y}-2 \epsilon_{z z}\right), \\
& =a\left(2-\frac{2 c_{12}}{c_{11}} \frac{d_{w}}{d_{w}+d_{b}}\right) \epsilon+\frac{b}{2}\left(1+\frac{2 c_{12}}{c_{11}} \frac{d_{w}}{d_{w}+d_{b}}\right) \epsilon,
\end{aligned}
$$

$$
\begin{aligned}
\left\langle 3 / 2,3 / 2\left|H_{\epsilon}\right| 3 / 2,-1 / 2\right\rangle & \\
= & \frac{\sqrt{3}}{2} b\left(\epsilon_{y y}-\epsilon_{x x}\right)=\frac{\sqrt{3}}{2} b\left(1+\frac{2 c_{12}}{c_{11}} \frac{d_{w}}{d_{w}+d_{b}}\right) \epsilon \\
& =\left\langle 3 / 2,1 / 2\left|H_{\epsilon}\right| 3 / 2,-3 / 2\right\rangle, \\
& =\left\langle 3 / 2,-3 / 2\left|H_{\epsilon}\right| 3 / 2,1 / 2\right\rangle, \\
& -\left\langle 3 / 2,-1 / 2\left|H_{\epsilon}\right| 3 / 2,3 / 2\right\rangle .
\end{aligned}
$$

We assume that the effect of strain on the conduction band is merely to shift the band edge, while the effective mass remains $m_{c}=0.067 m_{0}$, where $m_{0}$ is the free electron mass. Note that in Eqs. (4) and (5) the first term can be subtracted from the strain Hamiltonian since it is equivalent to a product of a constant term with the identity matrix. Just as the conduction band edge shift, this term affects only the value of the band gap, which is in any event established experimentally. Taking $c_{11}=1.188 \times 10^{12}$ dynes $/ \mathrm{cm}^{2}$, $c_{12}=0.538 \times 10^{12}$ dynes $/ \mathrm{cm}^{2}$, and $b=-1.5637 \mathrm{eV}$, we can obtain the numerical values for the elements of the strain Hamiltonian. These must be added to the Kohn-Luttinger Hamiltonian with the Luttinger parameters appropriate for

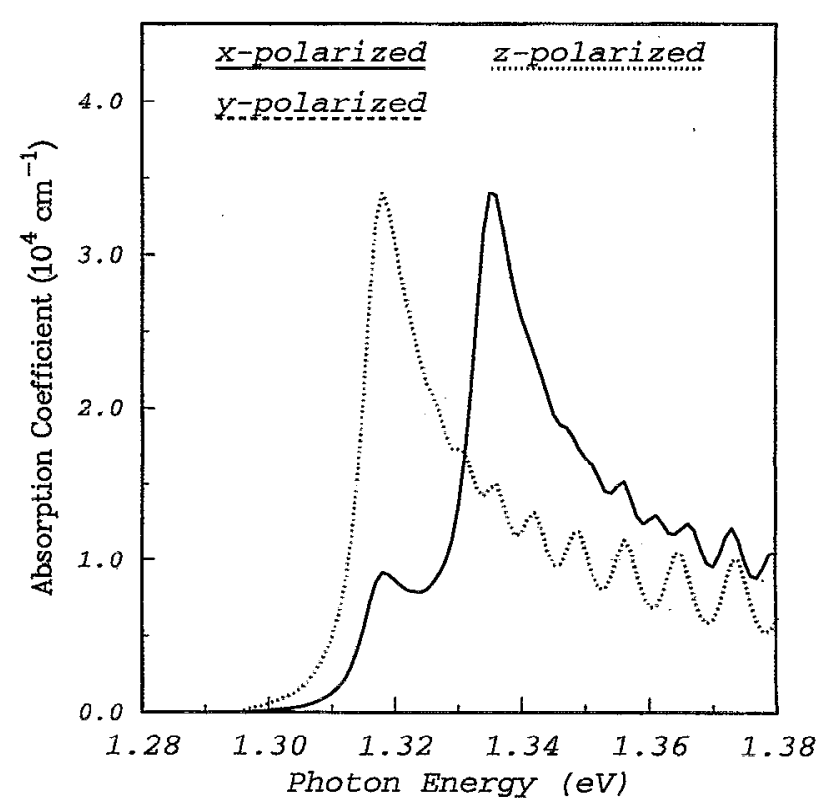

FIG. 1. The absorption coefficient for a $80 \times 80 \AA$ GaAs quantum wire near the band edge. All the strain tensor elements are zero.

the alloy in the active region and solved by discretizing spatial derivatives on a uniform mesh in the cross-sectional plane of the quantum wire. ${ }^{4}$ The absorption coefficient for the optical transitions in the quantum wire is given (in Gaussian units) by

$$
\begin{aligned}
\alpha(\hbar \omega)= & \frac{4 \pi^{2} e^{2} \hbar}{n_{0} c m_{0}^{2} W d_{w} \hbar \omega} \frac{2}{2 \pi} \int d k_{z} \sum\left|\boldsymbol{\epsilon} \cdot \mathbf{P}_{n m}\left(k_{z}\right)\right|^{2} \\
& \times \delta\left[E_{n}^{e}\left(k_{z}\right)-E_{m}^{o}\left(k_{z}\right)-\hbar \omega\right],
\end{aligned}
$$

where $\hbar \omega$ is the photon energy, $E^{e}$ and $E^{h}$ are the electron and hole energies, respectively, $n_{0}$ is the group refractive index, assumed constant for the considered energy range, $\epsilon$ is the unit polarization vector for the vector potential of incident light, $\mathbf{P}_{n m}$ is the momentum matrix element for the $n$th subband in the conduction band and the $m$ th subband in the valence band. The calculation of the momentum matrix element is outlined in Refs. 4, 7, and 8 . The Lorentzian broadening function is used in place of the delta function in determining the absorption coefficient which accounts for the uncertainty due to a finite lifetime of the electron and hole states. Another broadening function smoothens (insofar as it affects the band edge functional dependence) the discontinuities due to the use of a finite number of $k_{z}$ points in the numerical calculation of the band structure. The linewidths for the two functions are constant over the entire energy range and, thus, do not affect the ratios of different intensity peaks in the absorption spectrum.

\section{RESULTS}

We will compare our results for the strained system with the case of a GaAs quantum wire surrounded by $\mathrm{Al}_{0.3} \mathrm{Ga}_{0.7}$ As barriers. The absorption coefficient for the latter is shown in Fig. 1. Arguments based on the $\mathbf{k} \cdot \mathbf{p}$ theory ${ }^{6}$ in the spherical band structure approximation indicate the ratio of the matrix 


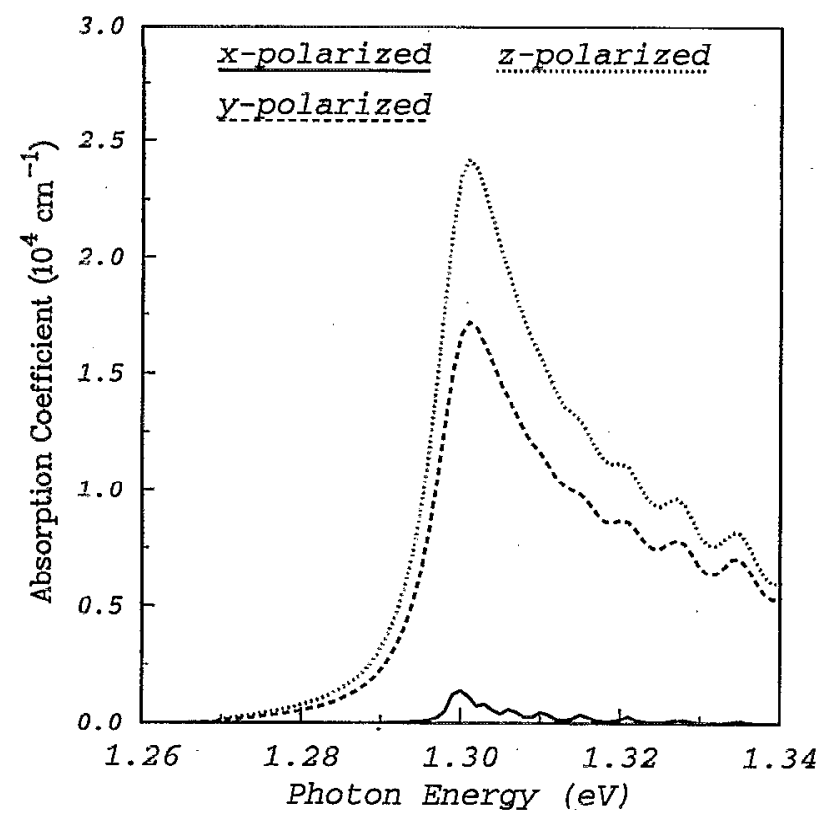

FIG. 2. The absorption coefficient for a $80 \times 80 \AA \mathrm{In}_{0.2} \mathrm{Ga}_{0,8}$ As quantum wire near the band edge $\left(\epsilon_{y y}=\epsilon_{z z} \approx-0.014, \epsilon_{x x} \approx 0.0032, r=0.25\right)$.

elements for light polarizations parallel and perpendicular to the wire axis is $4: 1$ at the band edge $\left(k_{z}=0\right)$. The relaxation of the assumption $\gamma_{2}=\gamma_{3}$ (where $\gamma_{i}$ are the Luttinger parameters) and the introduction of the Lorentzian broadening function in the expression for the absorption coefficient leads to a slight deviation of this rule since the close-lying second peak contributes to the absorption coefficient in the first. A small error may have also incurred by the discrete approximative nature of the solution. As a result, the ratio in Fig. 1 is 3.72:1. It is also predicted from the analytical arguments ${ }^{6}$ that the second peak in the absorption coefficient is solely due to transitions induced by light polarized perpendicular to the wire axis. This is verified by the detailed calculation in Fig. 1. The ratio between the absorption coefficient for the first and second peak are related to the ratios between the overlap integrals of the appropriate electron and hole states. For narrower wires, the overlap integral for the first transition is decreased in comparison to that for the second, and the amplitude of the second peak is enhanced relative to the first. The absolute value of the absorption coefficient is to some extent determined by the smoothing effect of the broadening functions which preserve only the area under the absorption curve. Slight irregularities in the absorption coefficient away from the band edge are due to the finite length of the quantum wire in the $z$ direction dictated by the use of a finite number of $k_{z}$ points.

By adding indium to the active region, it is possible to introduce biaxial compressive strain and to increase the separation between the first and second optical transitions. ${ }^{9}$ For a large compressive strain, the effects of lateral quantum confinement are entirely subsumed by the strain effects. The latter amount to a considerable suppression of the absorption strength for light polarized along the growth direction. ${ }^{10}$ In what follows we will focus on a $25 \%$ aspect ratio and later demonstrate that the effect of an increased aspect ratio is similar to that of increased strain albeit weaker. The absorption coefficient near the band edge is shown in Fig. 2 for an $80 \times 80 \AA \mathrm{In}_{0.2} \mathrm{Ga}_{0.8}$ As quantum wire. The ratio of the $z$-polarized to $y$-polarized light absorption coefficients is about 1.67:1, while the ratio of $z$-polarized to $x$-polarized light absorption coefficient is nearly 20:1. In the limiting case of the quantum well structure the $y$ and $z$ absorption coefficients are degenerate and the $x$ absorption coefficient is very small. Therefore, in spite of the modifications in the symmetry of the band edge states in the quantum wire by addition of $20 \%$ of In, polarization-resolved $\mathrm{PL}^{3}$ can still be used to estimate the extent of quantum confinement, even though the results should be treated with greater care. By increasing the lateral wire width $d_{w}$ to $200 \AA$, we found that the $z$ to $y$ ratio drops to $\approx 1.07: 1$. Table I contains the relative ratios of the polarization-dependent absorption coefficient at the band edge for a number of structures and material systems. By examining Eqs. (4) and (5) it is easy to establish that the effect of an increased aspect ratio is similar to and approximately half of the effect of an increased $\epsilon$ provided the change in the Luttinger parameters is small enough.

The behavior of the absorption coefficient is even more complex for a system with tensile strain. The absorption coefficient for the $80 \times 80 \AA \mathrm{GaAs}_{0.9} \mathrm{P}_{0.1}$ quantum wire is shown in Fig. 3. Although the energy separation between the first and second transition peaks is comparable to that in an unstrained quantum wire, considerable mixing of heavy- and light-holc states leads to enhanced absorption of $x$-polarized light and suppressed absorption of $y$-polarized light near the band edge. The trend in polarization dependence is thus approximately reversed compared with the case of compressive strain. A suppression of $x$-polarized light and an enhancement of $y$-polarized light absorption is observed for the sec-

TABLE I. Ratios of absorption coefficients for variously polarized light at the band edge, defined as the point at which the absorption coefficient for dominant polarization reaches its maximum for the first transition. For all wire structures cited, the aspect ratio $r=0.25$. Approximate values of the strain tensor elements are also shown.

\begin{tabular}{cccc}
\hline \hline Wire structure & $80 \times 80 \AA \mathrm{GaAs}$ & $80 \times 80 \AA \mathrm{In}_{0.1} \mathrm{Ga}_{0.9} \mathrm{As}$ & $80 \times 80 \AA \mathrm{In}_{0.2} \mathrm{Ga}_{0.8} \mathrm{As}$ \\
\hline$z: y: x$ ratio & $3.72: 1: 1$ & $19.57: 11.73: 1$ & $21.58: 15.29: 1$ \\
$\epsilon_{z z}: \epsilon_{y y}: \epsilon_{x x}$ & $0: 0: 0$ & $-0.007:-0.007: 0.0016$ & $-0.014:-0.014: 0.0032$ \\
Wire structure & $80 \times 200 \AA \mathrm{In}_{0.2} \mathrm{Ga}_{0.8} \mathrm{As}$ & $80 \times 80 \AA \mathrm{GaAs} \mathrm{s}_{0.9} \mathrm{P}_{0.1}$ & $80 \times 80 \AA \mathrm{GaAs} 0.8 \mathrm{P}_{0.2}$ \\
\hline$z: y: x$ ratio & $458.4: 427: 1$ & $5.74: 1: 3.09$ & $9.83: 1: 9.16$ \\
$\epsilon_{z z}: \epsilon_{y y}: \epsilon_{x x}$ & $-0.014:-0.014: 0.032$ & $0.0036: 0.0036:-0.0008$ & $0.0072: 0.0072:-0.0016$ \\
\hline \hline
\end{tabular}




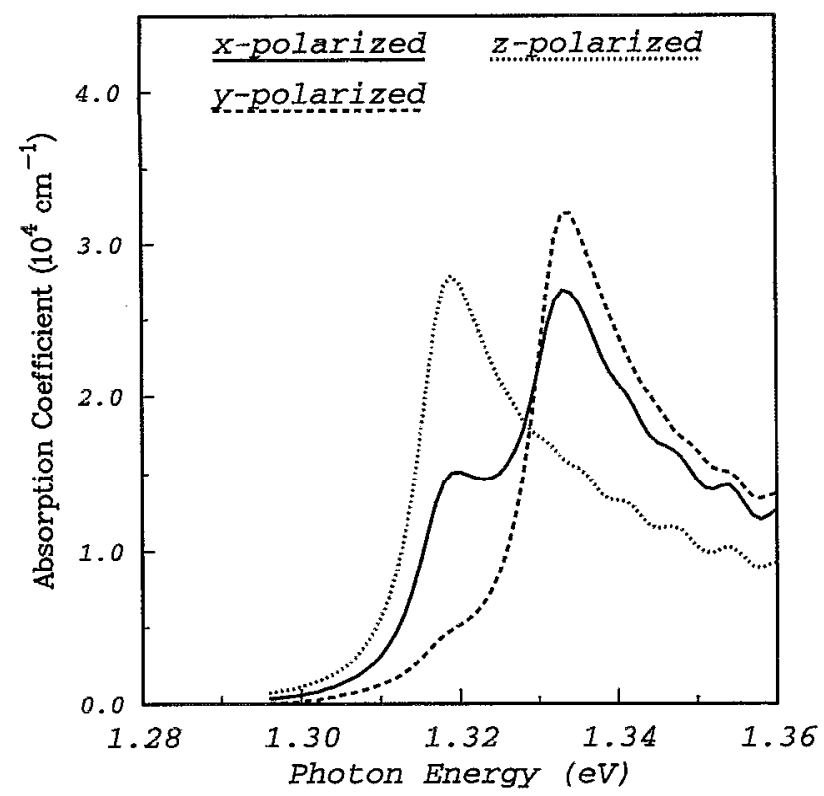

FIG. 3. The absorption coefficient for a $80 \times 80 \AA \mathrm{GaAs}_{0.9} \mathrm{P}_{0.1}$ quantum wire near the band edge $\left(\epsilon_{y y}=\epsilon_{z z} \approx 0.0036, \epsilon_{x x} \approx-0.0008, r=0.25\right)$.

ond transition peak. It can be seen by consulting Table $\mathrm{I}$, that for $20 \% \mathrm{P}$, the $x$ - and $z$-polarized light absorption strengths near the band edge are comparable, and $y$-polarized absorption is further suppressed. In the $20 \% \mathrm{P}$ system, the second transition accounts for some $z$-polarized light absorption in distinction to the previously considered cases.

\section{CONCLUSIONS}

Since, for practical purposes, the polarization dependence of the absorption coefficient is equivalent to the polar- ization dependence of PL spectroscopy, the results of this article can be applied to understand experimental data. The crucial lesson of this work is that built-in strain changes entirely the nature of the band edge states and the ratios between the absorption coefficients of variously polarized light. The polarization dependence of the absorption coefficient is intermediate between the cases of a quantum well structure with a similar strain perturbation, and a quantum wire without strain. This points to the possibility of using polarizationresolved $\mathrm{PL}$ as a tool for characterization of the degree of achieved quantum confinement and the quality of the quantum wire, ${ }^{4}$ albeit one that is not as powerful as in unstrained quantum wires owing to polarization discrimination weakened and blurred by strain.

\section{ACKNOWLEDGMENT}

This work was supported by the Army Research Office (URI program) under Grant DAAL03-92-G-0109.

'Y. Arakawa and H. Sakaki, Appl. Phys. Lett. 40, 939 (1982).

${ }^{2}$ Y. Arakawa, K. Vahala, and A. Yariv, Appl. Phys. Lett. 45, 950 (1984).

${ }^{3}$ J. Shuttlewood, O. Zia, K. Ko, P. Bhattacharya, S. W. Pang, I. Vurgaftman, J. Singh, and T. Brock, presented at 21 st International Symposium on Compound Semiconductors, San Diego, 1994.

${ }^{4}$ T. Tanaka, J. Singh, Y. Arakawa, and P. Bhattacharya, Appl. Phys. Lett. 62, 756 (1993).

${ }^{5}$ P. C. Sercel and K. J. Vahala, Appl. Phys. Lett. 57, 545 (1990).

${ }^{6}$ P. C. Sercel and K. J. Vahala, Phys. Rev. 44, 5681 (1991).

${ }^{7} \mathrm{~J}$. Singh, Physics of Semiconductors and Their Heterostructures (McGraw-Hill, New York, 1993), Chap. 7.

${ }^{8}$ I. Vurgaftman and J. Singh, IEEE J. Quantum Electron. QE-30, 75 (1994). ${ }^{9}$ G. C. Osbourn, J. Appl. Phys. 53, 1586 (1982).

${ }^{10}$ J. P. Loehr and J. Singh, IEEE J. Quantum Electron. 27, 708 (1991). 